\title{
CORRESPONDENCE \\ Long-term follow-up of recovered MPN patients with COVID-19
}

(c) The Author(s) 2021

Blood Cancer Journal (2021)11:115; https://doi.org/ 10.1038/s41408-021-00509-0

\section{Dear Editor,}

During the first wave of SARS-CoV-2 infection a European observational study was launched under the auspices of the European Leukemia Net (ELN), aiming at gathering information about the clinical epidemiology of COVID-19 in patients with chronic myeloproliferative neoplasms (MPN-COVID study).

Thirty-eight hematologic centers from Italy, Spain, Germany, France, United Kingdom and Poland, participated in the study and enrolled 180 consecutive patients with WHO-diagnosis of essential thrombocythemia (ET; $n=60)$, polycythemia vera (PV; $n=58$ ), prefibrotic-myelofibrosis (pre-PMF; $p=23$ ) and overt primary myelofibrosis (PMF; $n=39$ ) who developed COVID-19 from February 15 to May 31, 2020. During the acute phase of the infection, inhospital mortality affected almost $30 \%$ of 175 evaluable patients and the most vulnerable MPN subgroup was overt PMF (mortality 48\%) [1]. Another result deriving from this cohort concerned the thrombosis incidence, found significantly elevated in ET, where it reached almost $20 \%$ vs. $5 \%$ in PV and PMF, respectively [2].

At present, there is no information on the clinical condition of MPN patients discharged after COVID-19. Although SARS-CoV-2 infection has a variable clinical severity and mainly manifests itself as a respiratory syndrome, accumulating data revealed damage of hematopoietic system and vascular endothelium. This damage can persist even after the acute phase of infection [3]. Post COVID19 related consequences could be more frequent in clonal diseases such as MPN, whose natural history is marked by vascular complications and inherent risk of clonal evolution into myelofibrosis, myelodysplasia (MDS) and acute myeloid leukemia (AML).

In this paper, we report the events that occurred in 125 of the 175 patients $(71 \%)$, enrolled in MPN-COVID study, who survived to the acute phase of the infection. Participating centers were required to report in pre-established e-CRFs patient characteristics and outcomes collected after at least 6 months after COVID-19 infection recovery. The study was approved by the single center Ethical Committees.

\section{PATIENT CHARACTERISTICS \\ Before and at COVID-19}

In Table S1, surviving patients examined in the last-follow-up before COVID-19 (median: 1.4 months; interquartile range [IQR]: 0.8-3.0) presented blood counts and clinical picture consistent with the chronic phase of their respective MPN subtypes.

The great majority experienced an infection of moderate severity (94.4\%) and patients were managed at home $(n=38 ; 30.4 \%)$ or in regular wards $(n=80 ; 64 \%)$, whereas only seven $(5.6 \%)$ were in need of intensive care unit (ICU) admission. COVID-19 directed therapy included antiviral agents $(n=43 ; 35.8 \%)$ and in 28 patients $(23.7 \%)$ corticosteroids. High C-reactive protein (CRP), neutrophils on lymphocytes $(\mathrm{N} / \mathrm{L})$ ratio levels and $\mathrm{D}$-Dimer increase marked the clinical course. We point out that antithrombotic drugs including low molecular weight heparin (LMWH) and oral anticoagulants were prescribed in $54 \%$ and $2.5 \%$, respectively, of these 125 patients and that, in spite of this treatment, venous thromboembolism was diagnosed in 7 patients (5.6\%) and a stroke in a single case (0.8\%).

\section{Post-COVID}

The blood count values after 6 months from the resolution of the infection are presented in Table S1 as medians and interquartile ranges and do not show substantial alterations. Of note, chest CTscan was abnormal in $69 \%$ of 19 examined patients and in $10 \%$ patients the $\mathrm{O}_{2}$ saturation was less than 95\%. Among the laboratory tests, we noted the persistence of increased inflammation markers (i.e., $C R P \geq 0.8 \mathrm{mg} / \mathrm{dl}$ in $56 \%$ of cases; N/L ratio $\geq 3$ in $48 \%$; D-Dimer $\geq 500 \mathrm{ng} / \mathrm{mL}$ in $18 \%$ ), as reported in the general population after COVID-19 [4]. Cytoreductive therapy was used in $80 \%$ of cases and antithrombotic therapy included antiplatelet agents in $67.5 \%$ of cases and anticoagulants in $17.5 \%$.

\section{OUTCOMES POST-COVID-19 Symptoms}

A long-term follow-up study of these patients at 6 months post infection revealed ongoing symptoms in a third of the patients (Fig. S1). Among these, fever, cough, and dyspnea were the most commonly reported during the acute infection but largely remitted in the following 6 months. It has been reported that the persistence of symptoms is more frequent in patients over the age of 60 and in our cases, with a median of 70 years of age, it was also associated with persistence of inflammatory markers in more than half of them. Overall, these findings suggest a slow recovery after the acute phase of infection as also observed in other series of non-MPN patients $[5,6$,$] .$

\section{Major thromboses and bleedings}

Major thrombosis was registered in five patients (4\%); in one of them, massive fatal intestinal ischemia occurred (Table 1). None of these patients experienced thrombosis during COVID-19. The acute infectious disease of these patients was managed in the ordinary wards and, after discharge, antithrombotic therapy with LMWH was only used in a single patient who developed peripheral arterial thrombosis. Of the four patients receiving cytoreductive drugs, three were receiving ruxolitinib for PMF or ET, and the fourth was on hydroxyurea (HU). It is interesting to know that these events occurred after 5 months after the infection subsided, as it is well illustrated by the Kaplan Meyer thrombosis-free survival curve of Fig. 1A.

A single patient with PMF experienced a recurrence of gastrointestinal bleeding requiring blood transfusions $\sim 3$ months after discharge.

These findings are difficult to compare with the data reported in the general population in the post-infection period. A number of studies reported varying incidences of $1-5 \%$ of venous thromboembolism in patients after discharge, depending on the underlying disease, comorbidity, and concomitant antithrombotic prophylaxis [7-9]. Clearly, one factor that may explain some of these differences is 
Table 1. Main characteristics of patients with major outcomes at the 6-month follow-up after COVID-19 recovery.

\begin{tabular}{|c|c|c|c|c|c|c|}
\hline THROMBOSIS $(N=5)$ & Fatal event & MPN & Age & COVID-19 acute phase disposition & Cytoreduction & Anticoagulation \\
\hline Intestinal ischemia & Yes & MF & 71.7 & Regular ward & No & No \\
\hline Splenic infarction & No & MF & 72.3 & Regular ward & Ruxolitinib & No \\
\hline $\begin{array}{l}\text { Acute myocardial } \\
\text { infarction }\end{array}$ & No & PV & 80.6 & Regular ward & Hydroxyurea & No \\
\hline $\begin{array}{l}\text { Pheripheral arterial } \\
\text { thrombosis }\end{array}$ & No & MF & 75.4 & Regular ward & Ruxolitinib & Yes \\
\hline AML & Yes & MF & 49.3 & ICU & Hydroxyurea & 5.9 years \\
\hline AML & No & ET & 78.3 & Regular ward & Hydroxyurea & 6.0 years \\
\hline AML & No & $\begin{array}{l}\text { Pre- } \\
\text { PMF }\end{array}$ & 82.1 & Regular ward & Hydroxyurea & 3.8 years \\
\hline Non-Hodgkin lymphoma & No & ET & 60.0 & Regular ward & Hydroxyurea & 8.6 years \\
\hline $\begin{array}{l}\text { Progression of solid } \\
\text { cancer (parotid) }\end{array}$ & - & MF & 77.3 & Regular ward & Ruxolitinib & 21.7 years \\
\hline Suspected lung cancer & - & MF & 80.9 & Regular ward & unk & 10.9 years \\
\hline Multi Organ Failure & - & ET & 85.7 & Regular ward & Hydroxyurea & 5.8 years \\
\hline Thrombosis & - & MF & 71.7 & Regular ward & No & 10.2 years \\
\hline Heart failure & - & MF & 82.4 & Home & Unknown & 24.7 years \\
\hline Heart failure & - & $\begin{array}{l}\text { Pre- } \\
\text { PMF }\end{array}$ & 88.7 & Home & No & 8.8 years \\
\hline Unknown & - & ET & 87.0 & Regular ward & Unknown & 0.8 years \\
\hline
\end{tabular}

A

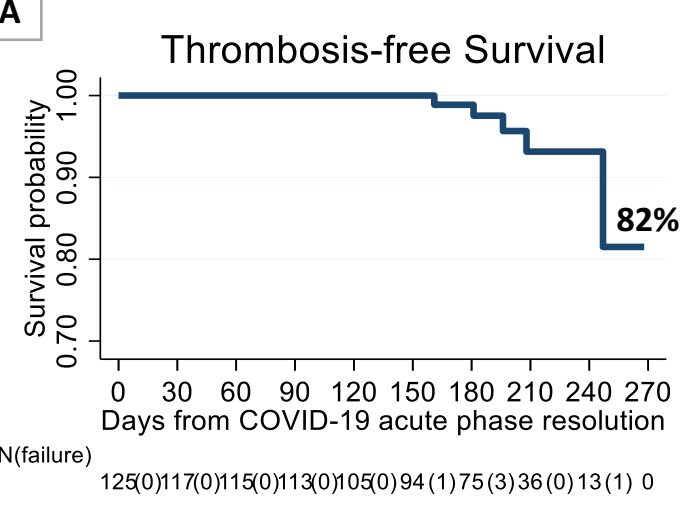

C

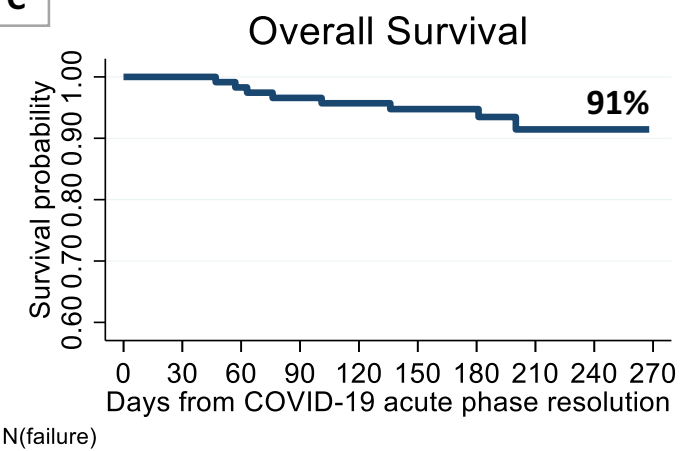

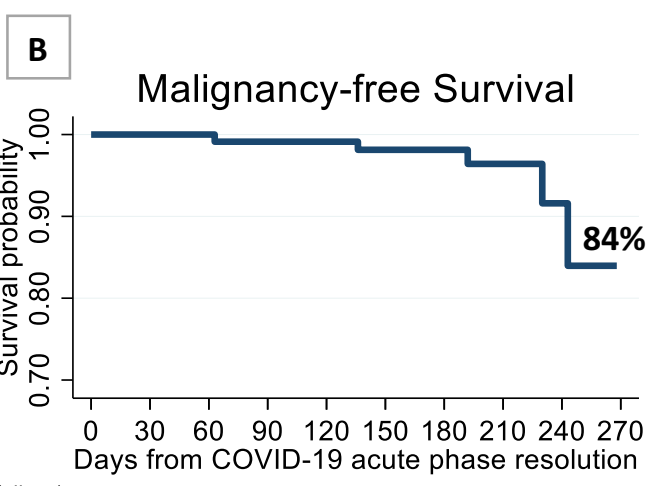
$\mathrm{N}$ (failure)

$125(0) 117(0) 115(1) 113(0) 105(1) 94(0) 75(1) 36(1) 13(1) 0$

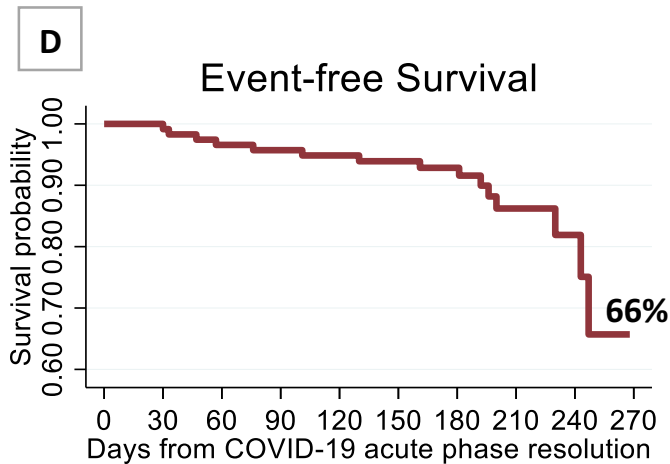
$\mathrm{N}$ (failure)

$125(0) 117(4) 113(1) 112(1) 104(1) 93(1) 74(4) 36(1) 13(2) 0$

Fig. 1 Major outcomes at 6-months follow-up after COVID-19 recovery. Kaplan-Meier survival estimates from COVID-19 recovery of (A) Thrombosis-free, (B) Malignancy-free, (C) Overall survival and (D) Event-free survival. 
the observation time after infection. In our cases with MPN, no event was recorded in the first 5 months but, instead, they occurred later, during the last period of our observation. Notably, our patients were not on LMWH prophylaxis during this time, which could possibly have reduced these vascular complications [10].

\section{Malignancies}

Acute myeloid leukemia. AML was diagnosed in 3 patients by morphology, immunophenotyping, cytogenetics and genetics including next generation sequencing (NGS) (Table S2).

Patient \#1, with PMF CALR-mutated, upon progression showed numerous recurrent karyotype abnormalities, and the presence of multiple genetic lesions typically associated with progression in AML was documented.

Patient \#2, with ET JAK2V617F (variant allele frequency [VAF] $31 \%)$, upon progression to AML showed a karyotype characterized by the presence of an additional marker, and genetic lesions associated with AML evolution were revealed by NGS.

Patient \#3, during chronic phase of pre-PMF, in addition to the MPL mutation (VAF 1\%) also showed the presence of high-risk genetic lesions [11]. On progression, a complex karyotype was found and the molecular profile documented additional genetic lesions of TP53 (VAF 91\%) and RUNX1 (VAF 44\%).

While it is conceivable that the COVID-19 hyperinflammatory state associated with the acute phase of the infection and persisting even after recovery could have accelerated disease progression in patient \#3, the molecular profile of the other two patients did not suggest such rapid progression to AML.

Large B-cell Non-Hodgkin lymphoma. Large B-cell non-Hodgkin lymphoma was diagnosed in a single case; the tumor developed predominantly in the brain and patient is currently alive on chemotherapy.

Parotid cancer. The patient had a rapid evolution post-COVID-19, whereas it was stable before. The tumor showed an unexpected aggressiveness leading to death of the patient.

These five malignant events were diagnosed as early as the second post-COVID-19 month and the probability of their occurrence was projected to $20 \%$ after 8 months. To our knowledge, the onset of neoplastic events in the immediate post-COVID-19 period in patients with MPN has not been reported so far. Given the low number of events, we were unable to investigate any risk factors. We can speculate that both MPN and COVID-19 share overlapping inflammatory mechanisms that may have favored the disease progression of malignant subclones already present in the chronic phase of MPN.

\section{Mortality}

Deaths occurred in eight patients after 9 months and the causes are listed in Table 1. Kaplan-Meier curve indicated that probability of death was $9 \%$ (Fig. 1C).

Event-free survival after 9 months, including freedom from thrombosis, malignancies and death, was $66 \%$ in the 125 surviving patients, who were followed-up for a median of 6 months postCOVID-19 recovery (Fig. 1D).

This multicenter European study, although with a relatively small number of patients, provides a descriptive analysis of MPN patients who survived after COVID-19. We here report a diversity of complications that further increase mortality and morbidity of MPN patients in the post-COVID-19 period. Indeed, $40 \%$ of these patients, when followed-up over 6 months after the acute COVID19 illness subsided, experienced both fatal and non-fatal events.

Our research indicates that the health consequences of COVID19 extend far beyond acute infection and suggest larger, multicenter analyses to augment and expand our observations. These signals should induce careful surveillance in all patients with MPN regardless of the severity of acute SARS-CoV-2 infection.
Tiziano Barbui $\mathbb{D D}^{1 凶}$, Alessandra lurlo $\mathbb{D D}^{2}$, Arianna Masciulli ${ }^{1}$, Alessandra Carobbio ${ }^{1}{ }^{1}$, Arianna Ghirardi ${ }^{1}$, Giuseppe Rossi ${ }^{3}$, Claire Harrison ${ }^{4}$, Alberto Alvarez-Larran ${ }^{5}$, Elena Maria Elli ${ }^{6}$, Jean-Jaques Kiladjian ${ }^{7}$, Mercedes Gasior Kabat ${ }^{8}$, Alberto Marin Sanchez ${ }^{9}$, Francesca Palandri (iD ${ }^{10}{ }^{\prime}$ Marcio Miguel Andrade-Campos ${ }^{11}$, Alessandro Maria Vannucchi ${ }^{12^{\prime}}$, Gonzalo Carreno-Tarragona (D) ${ }^{13}$, Petros Papadopoulos ${ }^{14}$, Keina Quiroz Cervantes (iD ${ }^{15}$, Maria Angeles Foncillas (D) ${ }^{16}$, Maria Laura Fox ${ }^{17}$, Miguel Sagues Serrano ${ }^{18}$, Elisa Rumi (1) ${ }^{19^{\prime}}$ Santiago Osorio ${ }^{20}$, Giulia Benevolo ${ }^{21}$, Andrea Patriarca (D) ${ }^{22^{\prime}}$ Begona Navas Elorza ${ }^{23}$, Valentin Garcia-Gutierrez ${ }^{24}$ Elena Magro Mazo ${ }^{25}$, Francesca Lunghi ${ }^{26}$, Massimiliano Bonifacio (iD ${ }^{27}$, Valerio De Stefano (D ${ }^{28}$ Juan Carlos Hernandez-Boluda (iD ${ }^{29}$, Emma Lopez Abadia ${ }^{30}$ ', Anna Angona ${ }^{31}$, Blanca Xicoy Cirici ${ }^{32}$, Marco Ruggeri ${ }^{33^{\prime}}$ Steffen Koschmieder (iD ${ }^{34}$, Marta Anna Sobas ${ }^{35}$, Beatriz Cuevas (iD ${ }^{36}$, Daniele Cattaneo ${ }^{2}$, Rosa Daffini ${ }^{3}$, Marta Bellini ${ }^{37}$, Natalia Curto-Garcia ${ }^{4}$, Marta Garrote ${ }^{5}$, Fabrizio Cavalca ${ }^{6}$, Lina Benajiba ${ }^{7}$, Beatriz Bellosillo ${ }^{11}$, Paola Guglielmelli (iD ${ }^{12}$, Oscar Borsani (D) ${ }^{19}$, Silvia Betti ${ }^{28}$, Silvia Salmoiraghi ${ }^{1,37}$ and Alessandro Rambaldi (ID $^{37,38}$ ${ }^{7}$ FROM Research Foundation, Papa Giovanni XXIII Hospital, Bergamo, Italy. ${ }^{2}$ Hematology Division, Foundation IRCCS Ca' Granda Ospedale Maggiore Policlinico, Milan, Italy. ${ }^{3}$ Spedali Civili, Brescia, Italy. ${ }^{4}$ Guy's and St. Thomas' NHS Foundation Trust, London, UK. ${ }^{5}$ Hospital Clinic de Barcelona, Barcelona, Spain. ${ }^{6}$ Hematology Division and Bone Marrow Transplant Unit. San Gerardo Hospital, ASST Monza, Monza, Italy. ${ }^{7}$ Hospital Saint-Louis, Paris, France. ${ }^{8}$ Hospital Universitario la

Paz, Madrid, Spain. ${ }^{9}$ Hospital General Universitario de Albacete, Albacete, Spain. ${ }^{10}$ IRCCS Azienda Ospedaliero-Universitaria di Bologna, Bologna, Italy. ${ }^{11}$ Hospital del Mar, Barcelona, Spain. ${ }^{12}$ Center Research and Innovation of Myeloproliferative Neoplasms (CRIMM), Department of Experimental and Clinical Medicine, Azienda Ospedaliera Universitaria Careggi, University of Florence, Florence, Italy. ${ }^{13}$ Hospital Universitario 12 de Octubre, Madrid, Spain. ${ }^{14}$ Hospital Clinico San Carlos, Madrid, Spain. ${ }^{15}$ Hospital Universitario de Mostoles, Madrid, Spain. ${ }^{16}$ Hospital Universitario Infanta Leonor, Madrid, Spain. ${ }^{17}$ Department of Hematology, Vall d'Hebron Institute of Oncology (VHIO), Vall d'Hebron Hospital Universitari, Vall d'Hebron Barcelona Hospital Campus, C/ Natzaret, 115-117, 08035 Barcelona, Spain. ${ }^{18}$ ICO L'Hospitalet-Hospital Moises Broggi, Sant Joan Despì, Barcelona, Spain. ${ }^{19}$ Department of Molecular Medicine, University of Pavia, Pavia, Italy. ${ }^{20} \mathrm{H} 30$,ospital Gregorio Maranon, Madrid, Spain. ${ }^{21} \mathrm{AOU}$ Città della Salute e della Scienza di Torino, Torino, Italy. ${ }^{22} \mathrm{AOU}$ Maggiore della Carità, Novara, Italy. ${ }^{23}$ Hospital Moncloa, Madrid, Spain. ${ }^{24}$ Hospital Ramon y Cajal, IRYCIS, Madrid, Spain. ${ }^{25}$ Hospital Universitario Principe de Asturias, Alcalà de Henares, Madrid, Spain.

${ }^{26}$ IRCCS Ospedale San Raffaele, Milano, Italy. ${ }^{27}$ Ospedale Policlinico "G.B. Rossi", Borgo Roma, Verona, Italy. ${ }^{28}$ Fondazione Policlinico "A. Gemelli" IRCCS, Rome, Italy. ${ }^{29}$ Hospital Clinico Universitario, INCLIVA,

Valencia, Spain. ${ }^{30}$ Hospital General de Elche, Elche (Alicante), Alicante, Spain. ${ }^{31}$ ICO Girona Hospital Josep Trueta, Girona, Spain.

${ }^{32}$ Hospital Germans Trias i Pujol, Badalona (Barcelona), Barcelona,

Spain. ${ }^{33}$ Ospedale San Bortolo, Vicenza, Italy. ${ }^{34}$ Department of Hematology, Oncology, Hemostaseology, and Stem Cell Transplantation, Faculty of Medicine, RWTH Aachen University, Aachen, Germany. ${ }^{35}$ Department of Hematology, Blood Neoplasms and Bone Marrow Transplantation, Wroclaw Medical University, Wrocław, Poland. ${ }^{36}$ Hospital Universitario de Burgos, Burgos, Spain.

${ }^{37}$ ASST Papa Giovanni XXIII, Bergamo, Italy. ${ }^{38}$ Università degli Studi di Milano, Milano, Italy. ${ }^{凶}$ email: tbarbui@fondazionefrom.it

\section{REFERENCES}

1. Barbui T, Vannucchi AM, Alvarez-Larran A, lurlo A, Masciulli A, Carobbio A, et al. High mortality rate in COVID-19 patients with myeloproliferative neoplasms after abrupt withdrawal of ruxolitinib. Leukemia. 2021;35: 485-93. 
2. Barbui T, De Stefano V, Alvarez-Larran A, lurlo A, Masciulli A, Carobbio A, et al. Among classic myeloproliferative neoplasms, essential thrombocythemia is associated with the greatest risk of venous thromboembolism during COVID-19. Blood Cancer J. 2021;11:21

3. Logue JK, Franko NM, McCulloch DJ, McDonald D, Magedson A, Wolf CR, et al. Sequelae in Adults at 6 Months After COVID-19 Infection. JAMA Netw Open. 2021;4:e210830.

4. Townsend L, Fogarty H, Dyer A, Martin-Loeches I, Bannan C, Nadarajan P, et al. Prolonged elevation of D-dimer levels in convalescent COVID-19 patients is independent of the acute phase response. J Thromb Haemost. 2021;19:1064-70.

5. Bellan M, Soddu D, Balbo PE, Baricich A, Zeppegno P, Avanzi GC, et al. Respiratory and psychophysical sequelae among patients with COVID-19 four months after hospital discharge. JAMA Netw Open. 2021;4:e2036142.

6. Nalbandian A, Sehgal K, Gupta A, Madhavan MV, McGroder C, Stevens JS, et al. Post-acute COVID-19 syndrome. Nat Med. 2021;27:601-15.

7. Salisbury R, lotchkova V, Jaafar S, Morton J, Sangha G, Shah A, et al. Incidence of symptomatic, image-confirmed venous thromboembolism following hospitalization for COVID-19 with 90-day follow-up. Blood Adv. 2020;4:6230-9.

8. Patell R, Bogue $T$, Koshy A, Bindal $P$, Merrill $M$, Aird WC, et al. Postdischarge thrombosis and hemorrhage in patients with COVID-19. Blood. 2020;136:1342-6.

9. Giannis D, S L Allen, J Tsang, S Flint, T Pinhasov, S Williams et al. Post-Discharge Thromboembolic Outcomes and Mortality of Hospitalized COVID-19 Patients: The CORE-19 Registry. Blood. Online ahead of print as https://doi.org/10.1182/ blood.2020010529. (2021).

10. Moores LK, Tritschler T, Brosnahan S, Carrier M, Collen JF, Doerschug K, et al. Prevention, diagnosis, and treatment of VTE in patients with coronavirus disease 2019: CHEST guideline and expert panel report. Chest. 2020;158:1143-63.

11. Tefferi A, Guglielmelli $P$, Lasho TL, Rotunno G, Finke $C$, Mannarelli $C$, et al. CALR and ASXL1 mutations-based molecular prognostication in primary myelofibrosis: An international study of 570 patients. Leukemia. 2014;28:1494-1500.

\section{ACKNOWLEDGEMENTS}

The study was supported by a research grant by the COVID " $3 \times 1$ project", BREMBO S. p.A., Bergamo, Italy (TB) and by AIRC $5 \times 1000$ call "Metastatic disease: the key unmet need in oncology" to MYNERVA project, \#21267 (MYeloid NEoplasms Research Venture AIRC). A detailed description of the MYNERVA project is available at https:// progettomynerva.it (AMV, PG). The study was also supported by HARMONY PLUS, which is funded through the Innovative Medicines Initiative (IMI), Europe's largest public-private initiative aiming to speed up the development of better and safer medicines for patients. The HARMONY Alliance has received funding from IMI 2 Joint Undertaking and is listed under grant agreement No. 945406. This Joint Undertaking receives support from the European Union's Horizon 2020 Research and Innovation Programme and the European Federation of Pharmaceutical Industries and Associations (EFPIA). IMI supports collaborative research projects and builds networks of industrial and academic experts in order to boost pharmaceutical innovation in Europe.

\section{AUTHOR CONTRIBUTIONS}

TB conceived and designed the study, supervised the analysis and wrote the paper. AMV, VDS, AR revised the study and contributed to manuscript writing. AM directed the project. $A C$ planned and performed statistical analyses. $A G$ contributed to dataset preparation. Al, CH, AAL, EE, JJK, MGK, AMS, FP, MMAC, AMV, GCT, PP, KSQC, MAF, MLF, MSS, ER, SO, GB, AP, BNE, VGG, EMM, FL, MB, VDS, JCHB, ES, BC, DC, RD, MB, NCG, MG, FC, LB, BB, PG, OB, SB, AR collected data. SS performed NGS analysis. All authors revised and approved the final version of the manuscript.

\section{COMPETING INTERESTS}

The authors declare no competing interests.

\section{ADDITIONAL INFORMATION}

Supplementary information The online version contains supplementary material available at https://doi.org/10.1038/s41408-021-00509-0.

Correspondence and requests for materials should be addressed to T.B.

Reprints and permission information is available at http://www.nature.com/reprints

Publisher's note Springer Nature remains neutral with regard to jurisdictional claims in published maps and institutional affiliations.

\section{(c) (i)}

Open Access This article is licensed under a Creative Commons Attribution 4.0 International License, which permits use, sharing, adaptation, distribution and reproduction in any medium or format, as long as you give appropriate credit to the original author(s) and the source, provide a link to the Creative Commons license, and indicate if changes were made. The images or other third party material in this article are included in the article's Creative Commons license, unless indicated otherwise in a credit line to the material. If material is not included in the article's Creative Commons license and your intended use is not permitted by statutory regulation or exceeds the permitted use, you will need to obtain permission directly from the copyright holder. To view a copy of this license, visit http://creativecommons. org/licenses/by/4.0/.

(c) The Author(s) 2021 